molar fraction of hydrogen ions without any large change in the actual degree of ionization.

6. A warning is given concerning the customary method of calculating the catalytic effect of un-ionized acid.

In conctusion the author wishes to express his hearty thanks to Professor H. A. Fales and Mr. J. C. Morrell for their courtesy in giving him access to unpublished work.

AMHERST, MASSACHUSETTS.

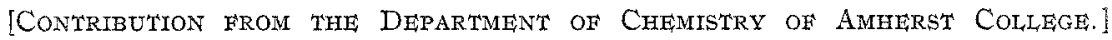

\title{
THE HYDRATION OF SUCROSE IN WATER SOLUTION AS CAL- CULATED FROM VAPOR-PRESSURE MEASUREMENTS.
}

By George Scatchard.

Received, July 27, 1921.

\section{Introduction.}

The determination of the existence of hydrates of sucrose in its aqueous solutions and the calculation of the average degree of hydration of the sucrose have been made from various properties of these solutions. Jones and Getman, ${ }^{1}$ Callendar, ${ }^{2}$ Washburn ${ }^{3}$ and Bousefield ${ }^{4}$ calculated the degree of hydration from the freezing point; Bousefield, Frazer and Myrick ${ }^{5}$ and Porter ${ }^{6}$ used the osmotic pressure; and Plilip, ${ }^{7}$ the solubility of hydrogen in sugar solutions. Einstein ${ }^{8}$ concluded from a kinetic treatment of viscosity that the sugar must be very heavily hydrated, but he did not determine the degree.

The calculation of hydration from the solubility of a gas depends on two assumptions: that the power to dissolve hydrogen of a given mass of water is independent of anything with which it may be mixed, and that the dissolving power of sucrose and of the hydrates of sucrose is zero. The principal justification for these assumptions is that, in the case of cane sugar and chloral hydrate, they give results in agreement with those from the colligative properties.

Washburn ${ }^{3}$ gives a very good review of hydration in solution, and notes that the colligative properties do not give mutually independent measures of the degree of hydration. The most rigorous treatment of either the

${ }^{2} \mathrm{H}$. C. Jones and F. H. Getman, Am. Chem. J., 32, 327 (1904),

${ }^{2}$ H. L. Callendar, Proc. Roy. Soc. (London), 80A, 466 (1900).

${ }^{8}$ E. W. Washburn, Technology Quart., 21, 376 (1908).

${ }^{4}$ W. R. Bousefield, J. Chem. Soc., 105, 600 (1914); Trans. Faraday Soc., 13, 141-55 (1917).

${ }^{5}$ J. C. W. Frazer and R. T. Myrick, This Jovrnal, 38, 1907 (1916).

${ }^{6}$ A. W. Porter, Trans. Faraday Soc., 13, 123 (1917).

7 J. C. Philip, J. Chem. Soc., 99, 711 (1907); Trans. Faraday Soc., 3, 140-5 (1907).

${ }^{8}$ A. Einstein, Ann. Phys., [4] 19, 301 (1906). 
osmotic pressure or the freezing point is to calculate the activity of the water by thermodynamic formulas and calculate the degree of hydration by assuming that this activity is proportional to the molar fraction of the water. That is, it is assumed that the solution is semi-ideal as defined in the previous paper. ${ }^{9}$ This is the method used by Callendar, by Washburn. and by Frazer and Myrick. Jones and Getman and Bousefield use the simpler method of calculating the hydration from the variation of the freezing point from Raoult's formula, which really involves the above assumption and in addition that of infinite dilution.

The assumptions made by Bousefield and by Porter in their treatments of osmotic pressure are quite different, but they both arrive at the formula $P(V-B)=R T$ as the exact one for osmotic pressure. Bousefield calculates the degree of hydration by taking $V-B$ as the volume of free water, with its density the same as that of pure water. Porter takes $B$ as the volume of the hydrate and assumes that the densities of the sugar and water in the hydrate are the same as those of the pure substances. When they both start from the same experimental results, ${ }^{10}$ Bousefield's calculations range from 85 to $95 \%$ of Porter's. Possibly most of this difference is due to different approximations of experimental values. We will not analyze their assumptions in detail since the same formula can be obtained from a great many different assumptions, and most of them must be wrong because they are mutually exclusive. But they all need the fundamental assumption that the forces of attraction are independent of the concentration, the condition which we found sufficient for semi-ideal solutions.

The exact calculation of the activity from the osmotic pressure requires a knowledge of the densities and the compressibilities of the solutions; the calculation from the freezing point requires the latent heat of fusion of ice and its temperature variation. The results are so uncertain that Washburn, Callendar and Frazer and Myrick do not attempt to calculate the degree of hydration exactly, but they assign different integral values to the hydration and calculate the corresponding freezing point or osmotic pressure to determine which value for the hydration agrees best with the experimental results. Washburn found that the results of several observers on rather dilute solutions were fitted by the assumption of a hexahydrate. Callendar used the measurements of Jones and Getman on more concentrated solutions and found that a pentahydrate gave more satisfactory results. Frazer and Myrick studied a much larger concentration range and found that a hexahydrate gave best agreement for the dilute solutions, a pentahydrate for intermediate concentrations and a tetrahydrate for the most concentrated solutions.

3 This Journai, 43, 2390 (1921).

10 Those of Morse and Frazer at 20\%. J. Chem. Soc., 105, 604 (1914); Trans. Faraday Soc., 13, 129 (1917). 
Vapor-pressure measurements have the advantage over the other colligative properties that they give the activity directly. They have the further advantage over the freezing point that they give results at constant temperature, and the effect of varying concentration can be determined directly. The accurate vapor-pressure measurements of Berkeley, Hartley and Burton ${ }^{11}$ at $0^{\circ}$ and $30^{\circ}$ give us the most exact means of determining the hydration of sucrose. They are used in the calculations of this paper.

\section{Analysis of Assumptions.}

Since we are to attempt to determine the degree of hydration with considerably greater accuracy than has been done hitherto, it is necessary to examine our assumptions rather closely. If the activity of the water is proportional to its molar fraction, the polarity or thermodynamic environment of the solution must be independent of the concentration. This means that the forces of attraction acting on any molecule must be independent of the kind of molecules surrounding it. A water molecule must have the same forces exerted on it when it is entirely surrounded by other water molecules as when it is entirely surrounded by sugar molecules or as when it is surrounded by any mixture of the two. These mutual forces depend upon the fields of force surrounding the individual molecules.

The field of force of a sugar molecule ought to be similar to that of a water molecule, or tather to a string of water molecules. The carbon atoms must be nearly strrounded by the hydrogen atoms and hydroxyl groups, and the sugar molecule ought to be represented by a nearly uniform field of force due to the hydrogen and hydroxyl radicals in equal numbers. But these are, of course, the same groups as those of the water molecules, and the fields of force of a stgar molecule ought to be very similar to that of the water molectles which it displaces in solution. Although these forces are very much greater than those in organic liquids, if they are the same for sugar and for water, aqueous sugar solutions ought to obey the laws of semi-ideal solutions as accurately as do mixtures of two hydrocarbons. Certainly, carbohydrates ought to obey these laws much more closely than most other solutes, especially ionogens, which have quite different force fields. It seems that we are justified in assuming that all the variation from ideality is due to chemical action and that the molar fraction of the water is proportional to its activity.

We must next consider the ways in which the number of molecules of each substance in solution may change from the number added. The dissociation of both water and sugar may certainly be neglected. We will assume that any association of sugar may also be neglected; this is fairly well justified since the molar fraction of sugar is always less than $12 \%$. Then there are but two kinds of chemical change possible: combination between two or more molecules of water, and between one of sugar and one or more of water. The extent of this latter reaction is what we are seeking to determine, and this makes it necessary to know the extent of the former. It is generally agreed that liquid water is partially associated into dihydrol $\left(\mathrm{H}_{2} \mathrm{O}\right)_{2}$ and perhaps a little trihydrol $\left(\mathrm{H}_{2} \mathrm{O}\right)_{8}$, and various calculations have been made of the extent of association. It seems that these are generally high, and that most of the properties which are customarily taken as evidence for association can be more simply accounted for by the larger forces of attraction between the molecules. However, it is a surprising fact that the assumption as to the degree of association of the water makes very little difference in the determination of the degree of hydration of the sucrose. Washburn

\footnotetext{
${ }^{11}$ Earl of Berkeley, E. G. J. Hartley and C. V. Burton, Phil. Trans., 218A, 295 (1919).
} 
and Callendar assume that there is no association, Frazer and Myrick assume that all the water exists as dihydrol, and their conclusions are not very different.

We will make our calculations on two assumptions, which seem the limits of probability. First, we will assume that there is no association; second, that the association is approximately that calculated by van Laar ${ }^{12}$ by the Ramsay-Ross Innes formula, 1.7 at $0^{\circ}$ and 1.6 at $30^{\circ}$ into dihydrol. That is, the molar fraction of monohydrol is 0.3 at $0^{\circ}$ and 0.4 at $30^{\circ}$. The degree of association will not be the same in the sugar solutions as in pure water but will obey the law of mass action. Also, in the case of association, the molar fraction of water will not equal its activity relative to that of pure water as unity, but the activity must be multiplied by the molar fraction of monobydrol in pure water.

Symbols.-For convenience of reference the symbols used in this paper are collected and defined here. We will let subscripts $w, 1,2$, s, anh and hyd refer to water, monokydrol, dihydrol, total sugar, anhydrous (unhydrated sugar) and hydrated sugar, respectively; $N$ is the molar fraction existing in solution; $N^{\prime}$, the molar fraction added; $N_{0}$, the molar fraction of monohydrol in pure water; $a$, the activity; $a_{w}$, the activity of monohydrol in terms of pure water as unity; $C$, the concentration of sucrose in grams per $100 \mathrm{~g}$. of water; and $m$ the number of molecules of water in one of sucrose hydrate.

Derivation of Formulas.-The calculation of the average degree of hydration is very simple for the case without association. From the number of grams of sugar added per $100 \mathrm{~g}$. of water we calculate the number of moles of water added to one mole of sugar,

$$
\frac{N_{\mathrm{w}}^{\prime}}{N_{\mathrm{s}}^{\prime}}=\frac{100 \times 342.24}{C \times 18.016}
$$

The number of moles of water existing in solution for each mole of sucrose is the ratio of their molar fractions; and, since $N_{\mathrm{w}}+N_{\mathrm{s}}=1$, then

$$
\frac{N_{\mathrm{w}}}{N_{\mathrm{s}}}=\frac{N_{\mathrm{w}}}{1-N_{\mathrm{w}}}=\frac{a_{\mathrm{w}}}{1-a_{\mathrm{w}}} \text {. }
$$

The moles of water which disappear from solution for each mole of sucrose, or the average degree of hydration of the sucrose, is obtained by subtracting (2) from (1).

For the case with association there is a little greater complexity. From the laws of semi-ideal solutions we have directly,

$$
N_{1}=N_{0} a_{\text {w }} \text {. }
$$

If the association follows the law of mass action in molar fractions,

$$
N_{2}=K N_{1}^{2}=K N_{\mathrm{o}}^{2} a_{\mathrm{w}^{2}}=\left(1-N_{\mathrm{o}}\right) a_{\mathrm{w}}{ }^{2} \text {. }
$$

The replacement of $K N_{0}{ }^{2}$ by $\left(1-N_{0}\right)$ is arrived at by solving the equation for the special case of pure water, for which $N_{2}=1-N_{0}$ and $a_{\mathrm{w}}=1$. The molar fraction of the sugar is equal to $1-N_{1}-N_{2}$. The quantity of water, calculated as moles of monohydrol, existing in solution per mole of sugar will be:

$$
\frac{N_{1}+2 N_{2}}{N_{\mathrm{s}}}=\frac{N_{1}+2 N_{2}}{1-N_{1}-N_{2}}
$$

The average degree of hydration is obtained by subtracting (5) from (1).

\section{The Measurements of Activity.}

For these calculations it is desirable to have as many and as accurate measurements of the vapor pressure as possible. In a concluding note Berkeley remarks that the $30^{\circ}$ values are slightly in error due to a small

${ }^{12}$ J. J. van Iaar, Z. physik. Chem., 31, 1 (1897). 
temperature gradient in the apparatus, and he records a few measurements with one apparatus where this error was corrected. In order to get the best values for all concentrations, a study was made of the difference between these corrected results and the uncorrected ones for each apparatus. This included the measurements on $\alpha$-methyl glucose and sulfuric acid. It was found that the average correction in the quantity $-\ln a_{w}$ was, within the experimental error, independent of the concentration and of the apparatus and equal to 0.00023 . So this correction was added to all the uncorrected results, and the best value was taken as the average of these values with those of the note, each weighted proportionally to the number of observations which it represents. Table I gives the best illus-

\begin{tabular}{|c|c|c|c|c|c|}
\hline \multicolumn{6}{|c|}{$T_{A B L E} I}$. \\
\hline $\begin{array}{l}\text { Cone. } \\
\text { G. per }\end{array}$ & & No. of & Not & No. of & Best Value. \\
\hline $100 \mathrm{~g}$ of $\mathrm{H}_{2} \mathrm{O}$. & +0.00023 & Determ. & $-\ln a_{\mathrm{w}}$. & Determ. & $-\ln a_{\mathrm{w}}$. \\
\hline 34.0 & 0.01927 & 5 & 0.01928 & 3 & 0.01927 \\
\hline 56.5 & 0.08406 & 2 & & 0 & 0.03406 \\
\hline 81.2 & 0.05233 & 4 & 0.05239 & 1 & 0.05234 \\
\hline 112.0 & 0.07739 & 3 & & 0 & 0.07739 \\
\hline 141.0 & 0.10282 & 2 & 0.10289 & 1 & 0.10284 \\
\hline 183.0 & 0.14208 & 9 & 0.14206 & 2 & 0.14208 \\
\hline 217.5 & 0.17563 & 1 & & 0 & 0.17563 \\
\hline
\end{tabular}

tration of the order of agreement. The measurements at $0^{\circ}$ are not affected by this correction.

\section{The Average Degree of Hydration.}

Table II gives the activity of the water and the degree of hydration of the sucrose calculated according to each of the two assumptions given above at $0^{\circ}$ and at $30^{\circ}$. It will be noticed that the effect of the assumption of

TABLE II.

Activity of Water and Average Degree of Hydration of Sucrose.

$0^{\circ}$.

$\begin{array}{rccc}\text { Conc. } & a_{\mathrm{w}}, & \begin{array}{c}\text { Av. hydrat. } \\ \text { without assoc. }\end{array} & \begin{array}{c}\text { Av. hydrat. } \\ \text { with assoc. }\end{array} \\ 34.0 & \ldots \ldots & \ldots . & \ldots . \\ 56.5 & 0.96545 & 5.68 & 6.08 \\ 81.2 & 0.94762 & 5.30 & 5.70 \\ 112.0 & 0.92327 & 4.93 & 5.32 \\ 141.0 & 0.89881 & 4.59 & 4.98 \\ 183.0 & 0.86320 & 4.07 & 4.45 \\ 217.5 & 0.83461 & 3.69 & 4.06 \\ 243.0 & 0.81330 & 3.46 & 3.82\end{array}$

$30^{\circ}$.

Av. hydrat. Av. hydrat. $a_{W^{-}}$without assoc. with assoc.

$\begin{array}{lll}0.98092 & 4.46 & 4.83 \\ 0.96651 & 4.76 & 5.13 \\ 0.94901 & 4.78 & 5.15 \\ 0.92553 & 4.53 & 4.89 \\ 0.90227 & 4.24 & 4.59 \\ 0.86755 & 3.83 & 4.17 \\ 0.83893 & 3.53 & 3.86 \\ \ldots \ldots & \ldots \ldots & \ldots .\end{array}$

association of the water is to increase the calculated value of the average hydration by about $4 / 10$ of a molecule, and that the increase is nearly independent of the concentration and temperature. The average degree 
of hydration decreases from about 6 to 3.5 , and it is smaller at $40^{\circ}$ than at $0^{\circ}$ as might have been expected. This agrees with the conclusions of Frazer and Myrick from the osmotic pressure. The decreasing values for the more dilute solutions at $30^{\circ}$ may be attributed to experimental error, for in the most dilute solutions an error corresponding to the correction made in Table I makes a difference of about 0.5 molecule in average hydration. This same explanation accounts for the results of Jones and Getman and of Porter on dilute solutions. Both find considerably larger values for dilute solutions; while Bousefield finds from the freezing-point measurements of Morse and Frazer that the values are irregular but always less than 6 . This means that values of the average degree of hydration calculated for solutions more dilute than $C=20$, at least from any measurements yet recorded, are of little value.

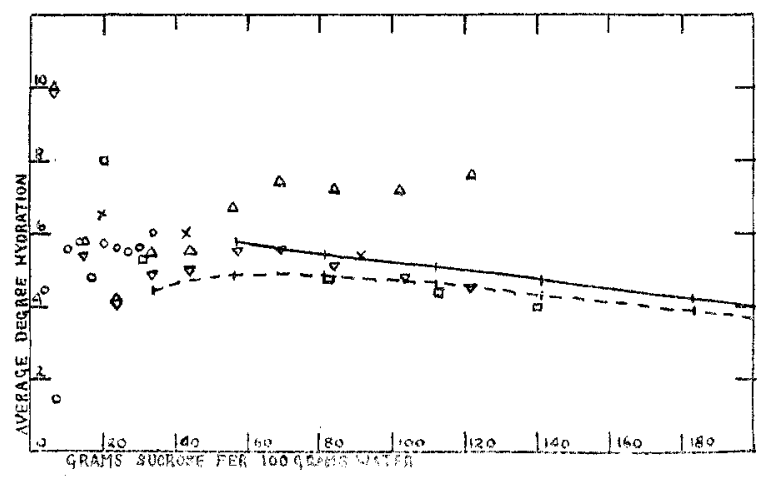

Fig. 1.-Average degree of hydration of sucrose.

SYMBOLS.

Full line: from vapor pressure at $0^{\circ}$, without association.

Dotted line: from vapor pressure at $30^{\circ}$, without association.

Triangles: from freezing point, Jones and Getman.

Inverted triangles: from freezing point, Jones and Getman, calculated by Scatchard.

Circles: from freezing point, Morse and Frazer, calculated by Bousefield.

Squares: from osmotic pressure at $0^{\circ}$, Berkeley, et al., calculated by Porter.

Crosses: from solubility of hydrogen at $18^{\circ}$, calculated by Philip.

Fig. 1 contains some of the more interesting determinations of the average degree of hydration. The abscissas are concentrations (g. of sucrose per $100 \mathrm{~g}$. of water), and the ordinates are the average degrees of hydration (molecules water for each molecule of sucrose). The values for the vaporpressure determinations assuming association of the water are omitted as they give lines parallel to those assuming no association and would only 
confuse the figure. Also, the figure does not extend to the most concentrated solutions for which the vapor pressures were measured. The increase of the hydration with increasing concentration found by Jones and Getman is seen to be due to the inaccuracy for concentrated solutions of their method of calculation. Their results as calculated by the author by the more accurate formula of Callendar ${ }^{13}$ decrease regularly when they pass the concentration where the experimental error is very large. All the methods appear to agree within their respective experimental errors, which are not large for the concentrated solutions. ${ }^{14}$

\section{The Nature of the Hydrate.}

We have already noted that the field of force of the sugar molecule is nearly uniform along its length. If there is a tendency for a water molecule to attach itself to one point in such a molecule, there should be a nearly

${ }^{13}$ Reference 2. p. 487, Formula VI.

${ }^{14}$ Note added November 2, -In the July number of This JournaL, (43, 1391-6 (1921)), Kendall, comes to the very interesting and important conclusion that, for sufficiently dilute solutions, the degree of association of the water does not affect the vaporpressure measurements, and that it may be neglected in calculating the degree of hydration of the solute from the colligative properties.

The present paper gives an interesting supplement to his conclusions in showing the magnitude of the effect for solutions which are not ordinarily considered as dilute. The weight-molar concentration varies from $1 M$ to $7 M$. Since the calculations from the data on hand are relatively simple, the average degree of hydration was also calculated with the assumption that $N_{0}=0.01$ and 0.001 , that is that in pure water only one molecule in every hundred and in every thousand, respectively, is monohydrol, while the others are dihydrol. The two calculations agree within 0.01 molecule in every case, so that only those for $N_{0}=0.001$ are tabulated. The differences between these values and those for $N_{0}=1.0$, or for no association, (from Table II) are also given.

TABLE II A.

AvERAGE DEGREE OF HXDRATION OF SUCROSE, AsSUMING $N_{0}=0.001$. $0^{\circ}$.

$\begin{array}{rcccc}\text { Conc. } & \text { Av. hydrat. } & \begin{array}{c}\text { Diff. from } \\ \text { no. Assoc. }\end{array} & \text { Av. hydrat. } & \begin{array}{r}\text { Diff. from } \\ \text { no. Assoc. }\end{array} \\ 34.0 & \ldots \ldots & \ldots . & 4.97 & 0.51 \\ 56.5 & 6.17 & 0.49 & 5.25 & 0.49 \\ 81.2 & 5.79 & 0.49 & 5.27 & 0.49 \\ 112.0 & 5.41 & 0.48 & 5.01 & 0.48 \\ 141.0 & 5.06 & 0.47 & 4.71 & 0.47 \\ 183.0 & 4.53 & 0.46 & 4.29 & 0.46 \\ 217.5 & 4.14 & 0.45 & 3.98 & 0.45 \\ 243.0 & 3.91 & 0.45 & \ldots \ldots & \ldots .\end{array}$

In this concentration range, these widely different assumptions make a difference of approximately one-half molectle in the average hydration. Comparison with Fig. 1 will indicate the unimportance of this compared to the experimental error in dilute solutions. Whether this factor need be considered in any case depends, of course, upon the accuracy of the results desired and justified by the experimental accuracy and the validity of the laws of semi-ideality for the case in question. 
equal tendency toward attachment at several other points. Probably the addition of a water molecule at one point will not change the field or tendency to hydrate at other points. Then that hydrate in which all the equivalent positions are filled ought to be very much more stable than any of the lower hydrates. This hydrate will also have a nearly uniform field and may have a tendency to hydrate further. If so there will be another higher hydrate, existing in smaller quantities than the first, but larger than any of the other hydrates.

We will assume that there is one such hydrate of so great a relative stability that the quantities of all the other hydrates are negligibly small, and that the whole system can be cousidered as an equilibrium between water, anhydrous sugar and this hydrate. If the solution is semi-ideal the formation of this hydrate will obey the law of mass action in terms of molar fractions. By application of the law we can determine how well the assumption of any hydrate will explain the experimental facts.

It must be emphasized that this theory of one rather complex hydrate is not applied to hydration in solution in general, but is limited to carbohydrates, whose large uniform molecules should give them very special properties. This explanation of a varying average degree of hydration is believed to be original; and, although the literature contains many statements that hydrate formation obeys the law of mass action, there seems to have been no previous attempt to test with the experimental results.

Consider first the case in which the water in not associated. The law of mass action for the reaction $\mathrm{C}_{22} \mathrm{H}_{22} \mathrm{O}_{11}+m \mathrm{H}_{2} \mathrm{O} \rightleftharpoons \mathrm{C}_{12} \mathrm{H}_{22} \mathrm{O}_{11} \cdot m \mathrm{H}_{2} \mathrm{O}$ will be

$$
\frac{N_{\text {hyd }}}{N_{\text {anh }} \times\left(N_{\mathbf{w}}\right)^{m}}=K_{\mathrm{m}} \text {. }
$$

We know $N_{\mathrm{w}} / N_{\mathrm{s}}$ and the average degree of hydration. Since $N_{\mathrm{w}}+N_{\mathrm{s}}=1$,

$$
1+\frac{N_{\mathrm{w}}}{N_{\mathrm{s}}}=\frac{1}{N_{\mathrm{s}}} \text {. }
$$

The average degree of hydration divided by $m$ will be

$$
\frac{N_{\text {hyd }}}{N_{\mathrm{s}}} \text {, and } 1-\frac{N_{\text {hyd }}}{N_{\mathrm{s}}}=\frac{N_{\mathrm{anh}}}{N_{\mathrm{s}}}
$$

From these ratios the calculation of the molar fractions and of $K$ is direct and simple.

For the case in which the water is associated, we have already determined $N_{1}$, which must replace $N_{\mathrm{w}}$ in the formula, and $N_{\mathrm{s}}$ in calculating the average degree of hydration. Dividing this average degree of hydration by $m$ and multiplying by $N_{\mathrm{s}}$ will give $N_{\text {hyd }}$, and $N_{\text {anh }}=N_{\mathrm{s}}-N_{\text {hyd }}$. This gives us all the data necessary for calculating $K_{m}$ by Equation 6 . Table III contains the values of $K$ for $m=6$ and $m=7$. The choice must lie between these two, for the values of $K_{8}$ decrease too rapidly to be possible 
TABLE III.

EQUilibrium CONSTANTS For Hydration OF SUCROSE. Without association.

\begin{tabular}{|c|c|c|c|c|c|c|c|c|}
\hline & With & $t$ asso & tion. & & & ith as & ation. & \\
\hline & & & & $0^{\circ}$. & & & 30 & \\
\hline Conc. & $K_{6}$ & $K_{7}$. & $K_{8_{0}}$ & $K_{7}$. & $K_{8}$. & $K_{7}$. & $K_{8}$ & $K \eta$ \\
\hline 34.0 & & & 3.25 & 2.01 & $\ldots$ & $\ldots$ & 1.14 & 1.56 \\
\hline 56.5 & 21.76 & 5.49 & 4.72 & 2.70 & absurd & 38.76 & 1.76 & 2.12 \\
\hline 81.2 & 10.52 & 4.56 & 5.38 & 3.11 & 36.22 & 29.28 & 2.02 & 2.45 \\
\hline 112.0 & 7.42 & 4.16 & 4.92 & 3.16 & 17.35 & 25.33 & 1.71 & 2.43 \\
\hline 141.0 & 6.18 & 4.02 & 4.47 & 3.16 & 12.66 & 23.74 & 1.48 & 2.39 \\
\hline 183.0 & 5.10 & 3.89 & 4.14 & 3.27 & 9.50 & 22.32 & 1.31 & 2.44 \\
\hline 217.5 & 4.72 & 3.95 & 4.09 & 3.47 & 8.48 & 22.35 & 1.26 & 2.57 \\
\hline 243.0 & 4.71 & 4.16 & $\ldots$ & $\ldots$ & 8.33 & 23.39 & $\ldots$ & , \\
\hline
\end{tabular}

With association.

and $K_{5}$ leads to absurd results. The values of $K_{6}$ are very good for the most concentrated solutions, but they increase rapidly with dilution for the more dilute; the values for $K_{7}$ are fair throughout. At $30^{\circ}$ either gives good constancy. The results for the dilute solutions are much less important, for there a small error in the vapor pressure corresponds to a large change in $K$. To illustrate this and to give a better test of the agreement of the theory with experiment, calculations are given in Table IV of the values of the vapor pressure which give constant values of $\dot{K}$ for the case without association.

These calculations have to be made by trial and error and are rather tedious. The values selected for the constants are approximately those which give the smallest deviations from the experimental results, but the choice may be more fortunate in one case than in another. So the results for $K_{6}$ and $K_{7}$ must not be compared too closely. At $0^{\circ}$, however, the heptahydrate clearly gives much better agreement. The table shows the enormous effect in $K$ of a small error in the vapor-pressure measurements for the dilute solutions.

\section{TABLE IV.}

Values of Vapor Pressure Which Give Constant values for $K$ (Water Not Associatep).

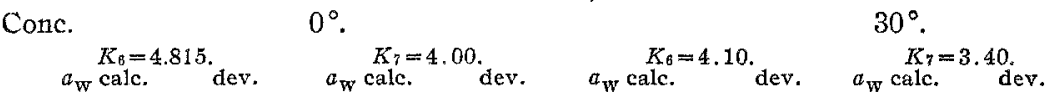

$34.0 \ldots \ldots \ldots \ldots \ldots \ldots \ldots \ldots \ldots \ldots \ldots .6 .98083-0.000090 .98064-0.00028$

$56.50 .96649+0.001040 .96589+0.000440 .96667+0.000160 .96614-0.00037$

$81.20 .94930+0.001680 .94808+0.000460 .94973+0.000720 .94867-0.00034$ $112.0 \quad 0.92565+0.002380 .92359+0.000320 .92663+0.001100 .92492-0.00061$

$141.00 .90145+0.002640 .89888+0.000070 .90322+0.000950 .90122-0.00105$ $183.00 .86441+0.001210 .86251-0.000690 .86777+0.000220 .86658-0.00097$ $217.5 \quad 0.83399-0.000620 .83415-0.000460 .83883-0.000100 .83959+0.00066$ $243.00 .81250-0.000800 .81482+0.00152 \ldots \ldots \ldots \ldots \ldots \ldots \ldots \ldots \ldots$

\section{The Activity of the Sucrose.}

It is possible to attack the constitution of the hydrate from another 
point of view. By the use of Lewis and Randall's modification ${ }^{15}$ of the Duhem-Margules equation for the dependence of the activity of one component upon that of the other, it is possible to calculate the change in the activity of the sucrose with changing concentration. The method of calclilation is to plot $\log \left(a_{\mathrm{w}} / N_{\mathrm{w}}^{\prime}\right)$ against $N_{\mathrm{w}}^{\prime} / N_{\text {anl }}^{\prime}$ (abscissa) and to measure the area under the curve between the concentrations chosen. This gives the change in $\log \left(a_{\mathrm{anh}} / N_{\mathrm{anh}}^{\prime}\right)$ for it is the anhydrotıs sugar which is added to the solution. If our theory is correct $a_{\text {anh }} / N_{\text {anh }}^{\prime}=$ $N_{\text {anh }} / N_{\text {anh }}^{\prime}$. With solutions of such strength as we are using it is unsafe to extrapolate in either direction. The simplest method is to make the comparison directly between the change in $\log \left(a_{\text {anh }} / N_{\text {anh }}^{\prime}\right)$ and that in $\log \left(N_{\text {anh }} / N_{\text {anh }}^{\prime}\right)$ as calculated according to the various assumptions. Since the values of the latter quantity are more accurate for the bigher concentrations, the change is calculated from the highest concentration.

Table $\mathrm{V}$ gives the values of $N_{\mathrm{w}}^{\prime} / N_{\mathrm{anb}}^{\prime}, \log \left(a_{\mathrm{w}} / N_{\mathrm{ww}}^{\prime}\right)$ and the increase of. $\log \left(a_{\mathrm{anh}} / N_{\mathrm{anh}}^{\prime}\right)$ at $0^{\circ}$ and at $30^{\circ}$. Table VI gives the values of the change in $\log \left(N_{\mathrm{anh}} / N_{\mathrm{anh}}^{\prime}\right)$ as calculated according to the different assumptions and the differences between these values and the observed

TABLE V.

Actrvity of Sucrose by DUHEM-Margules Equation.

$0^{\circ}$. $30^{\circ}$.

\begin{tabular}{|c|c|c|c|c|c|}
\hline Conc. & $\frac{N^{\prime} w}{N_{\text {ant }}^{\prime}}$. & $\log _{\frac{\mathrm{N}_{\mathrm{w}}^{\prime}}{\mathrm{w}^{\prime}}}$. & $\Delta \log \frac{a_{\text {anh }}}{N_{\text {anh }}^{\prime}}$. & $\frac{a_{\mathrm{w}}}{\log \frac{N_{W}^{\prime}}{N_{w}}}$. & $\Delta \log _{N_{\text {anh }}^{\prime}} \frac{a_{\text {anh }}}{N^{\prime}}$ \\
\hline 34.0 & 55.872 & & $\ldots \ldots$ & -0.00066 & -0.4520 \\
\hline 86.5 & 33.622 & -0.00254 & -0.4679 & 0.00206 & 0.3914 \\
\hline 81.2 & 23.395 & 0.00519 & 0.3946 & 0.00456 & 0.3237 \\
\hline 112.0 & 16.961 & 0.00979 & 0.3035 & 0.00873 & 0.2402 \\
\hline $14: 1.0$ & 13.473 & 0.01524 & 0.2218 & 0.01357 & 0.1668 \\
\hline 183.0 & 10.381 & 0.02394 & 0.1198 & 0.02176 & 0.0705 \\
\hline 217.5 & 8.734 & 0.03144 & 0.0494 & 0.02920 & 0.0000 \\
\hline 243.0 & 7.818 & 0.03747 & 0.0000 & & \\
\hline
\end{tabular}

TABLE VI A.

Comparison of Activity and Molar Fraction of Sucrose. (Without Association). $0^{\circ}$.

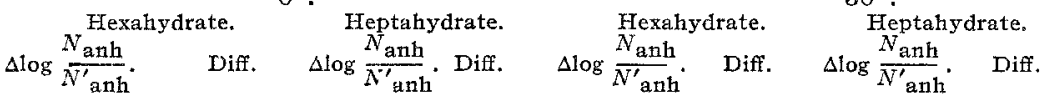

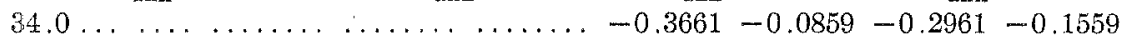

$56.5-1.0352+0.5673-0.5662+0.0983 \quad 0.4319+0.0405 \quad 0.3221-0.0693$

$\begin{array}{llllll}81.2 & 0.6720+0.2774 & 0.4294+0.0348 & 0.4088+0.0851 & 0.2957 & -0.0280\end{array}$

$\begin{array}{lllllll}112.0 & 0.4515+0.1480 & 0.3096+0.0061 & 0.2960+0.0558 & 0.2178 & -0.0224\end{array}$

$\begin{array}{lllllll}141.0 & 0.3064+0.0846 & 0.2177 & -0.0041 & 0.1929+0.0261 & 0.1448 & -0.0220\end{array}$

$\begin{array}{lllllll}183.0 & 0.1435+0.0237 & 0.1063 & -0.0135 & 0.0743+0.0038 & 0.0571 & -0.0134\end{array}$

$\begin{array}{lllllll}217.5 & 0.0501+0.0007 & 0.0384 & -0.0110 & 0.0000 \ldots \ldots & \ldots .0000 \ldots \ldots\end{array}$

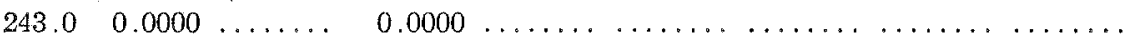

${ }_{15}$ G. N. Lewis and M. Randall, ThIs Journal, 43, 233 (1921). 
TABLE VIB.

(With Associatron.)

\begin{tabular}{|c|c|c|c|c|c|c|c|c|}
\hline 34.0 & & & & & -0.3990 & -0.0530 & -0.2968 & -0.1552 \\
\hline 56.5 & & & -0.6493 & +0.1814 & 0.4992 & +0.1078 & 0.3340 & -0.0574 \\
\hline 81.2 & -0.9480 & +0.5534 & 0.4734 & +0.0788 & 0.4816 & +0.1579 & 0.3109 & -0.0128 \\
\hline 112.0 & 0.5621 & +0.2586 & 0.3331 & +0.0296 & 0.3396 & +0.0994 & 0.2269 & -0.0133 \\
\hline 1.0 & 0.3624 & +0.1406 & 0.2304 & +0.0086 & 0.2152 & +0.0484 & 0.1489 & -0.0179 \\
\hline 3.0 & 0.1614 & $\begin{array}{r}+0.0416 \\
\end{array}$ & 0.1096 & -0.0102 & 0.0806 & +0.0101 & 0.0575 & -0.0130 \\
\hline 7.5 & 0.0549 & +0.0055 & 0.0387 & -0.0107 & 0.0000 & & 0.0000 & \\
\hline 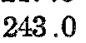 & 0.0000 & & 0.0000 & & & & & \\
\hline
\end{tabular}

changes in $\log \left(a_{\mathrm{anh}} / N_{\mathrm{anh}}^{\prime}\right)$. Again the values for the more concentrated solutions agree better for the hexahydrate, while over the greater part of the range they fit the heptahydrate better. And again, for the more concentrated solutions, there is a variation in the opposite direction for these two hydrates, showing that higher or lower hydrates would give poorer agreement.

These results are important as showing that the activity of the sucrose varies approximately as it should if our theories are correct. They confirm the idea that a varying proportion of the sugar exists in the unhydrated condition. As a test between the different assumptions, however, they are not entirely independent of the calculations of the equilibrium constants. If we use the vapor pressures which give constant values of $K_{6}$, the variation in $\log \left(a_{\mathrm{anh}} / N_{\mathrm{anh}}^{\circ}\right)$ will agree exactly with that of $\log$ $N_{\text {anb }} / N_{\text {anh }}^{\prime}$, and similarly for the heptahydrate.

\section{Interpretation of the Results.}

The vapor-pressure measurements do not agree within their apparent experimental error with the existence of either a hexahydrate or a heptahydrate alone, and our results seem to indicate that no assumption concerning the association of the water will better the agreement. It is possible that there is a constant experimental error in the vapor-pressure measurements which is larger than the agreement of the individual experiments indicates. An error of $0.1 \%$ at $30^{\circ}$ and of $0.2-0.3 \%$ at $0^{\circ}$ would account for the variation either for the hexahydrate or for the heptahydrate, depending upon the direction of the deviation. This is, however, from 5 to 10 times the apparent experimental error.

There seem to be three other possible explanations. First, our solutions may not be strictly semi-ideal, and the deviation from proportionality between the activities and the molar fractions might explain the results for either the hexahydrate or the heptahydrate, depending again upon the direction of the deviation. Second, our assumption of one hydrate so much more stable than the others may be entirely wrong, and the solution may contain an equilibrium mixture of a large number of hydrates in nearly equal quantities. Third, our assumption of one hydrate may be 
only slightly in error, and there may be but one higher hydrate as suggested earlier in this paper. If so the predominating hydrate must be the hexahydrate or possibly an even lower one. If a hexahydrate, the agreement of the concentrated solutions would be due to the fact that the second hydrate would exist only in very small quantities in these solutions. The increasing quantities on dilution would account for the increase of $K$ when calculated for the hexahydrate alone. Doubtless, given an arbitrary choice of the composition of this hydrate and its constant of dissociation, it would be possible to fit the experimental results within their error of measurement. But the formula would involve four arbitrary constants, and its agreement would not give a very strong confirmation of the theory. At $30^{\circ}$ this higher hydrate would exist in much smaller quantities than at $0^{\circ}$.

Evidence from other Sources.-The surest proof of the existence of a compound in solution is its separation as a solid phase, identified by anal$y$ sis or by the freezing-point curve. Guthrie ${ }^{16}$ mentions a cryohydrate separating at $-8.5^{\circ}$ with a composition of 5.27 mole per cent. $(51.4 \%)$ sugar. This is far off the curve for anhydrous sugar and, if it were real, would indicate a hydrate. However, the ice curve drawn from Guthrie's other measurements will not pass through this point without sharp curvature. Jones and Getman obtained a smooth curve up to a concentration of 6.43 mole per cent. which freezes at $-9.13^{\circ}$. The author has made preliminary measurements extending to 8 mole per cent. where the freezing point is about $-13^{\circ}$. This extends the ice curve slightly beyond the sugar-solubility curve. In no case did anything but ice separate and the curve seems entirely regular. The fact that only ice separated in these experiments does not prove, of course, that the system ice: solution is not a metastable one from which a hydrate might be separated. The study of the freezing-point curve will be continued, and will at least extend the ice curve to meet that of sugar. So the present evidence from the phase rule means nothing. The molar fraction of the hydrate is small even at its highest concentration, and it should be so similar in polarity to the other components of the solution that it ought to be quite soluble. Then failure to isolate a solid hydrate would not be unexpected.

All of the physical properties of sucrose solution which have been measured seem to obey the additive law so closely that they give no indication except that there is a slight variation from ideality, but not necessarily from semi-ideality.

If the degree of association of water were accurately fixed, some insight could probably be gained from the heat of dilution of sucrose solutions. But the heat of reaction calculated from the change in the equilibrium constant with the temperature would depend very largely upon the assumption as to the degree of ionization of the water. There would also

${ }^{16}$ F. Guthrie, Phil. Mag., [5] 2, 216 (1876). 
be the accompanying reaction of the association of the water with a large thermal effect.

The only evidence other than that from the colligative properties seems to be that from the speed of inversion. It has been shown in the preceding paper that probably 6 , but possibly 5 or 7 water molecules enter into the reaction with each sugar molecule, and that the most probable explanation is a preliminary hydration of the sugar molecule. The assumptions underlying the speed of inversion method are almost entirely different from those of the present paper. It is true that the assumption. of semi-ideal solution was made in changing from activity of sucrose to molar fraction. Also the justifiability of that assumption is confirmed by its success in accounting for the variation of hydrogen-ion activity on the addition of sugar. Our only other assumptions, except for small correcting terms which might have been neglected, are that the speed of reaction in solutions is proportional to the activities of the reactants, and that the activity of the hydrogen ion which determines its catalytic effect is the same as that which determines the electromotive-force measurements.

The determination from the vapor pressures assumes that the solutions are semi-ideal, and that there is one hydrate which is very much more stable than the others. The hypothesis that the formation of the hydrate obeys the law of mass action in terms of molar fractions follows as a thermodynamic necessity from the assumption of semi-ideality. We have two independent methods which lead us to the conclusion that aqueous sugar solutions are equilibrium mixtures of sucrose, water and a hydrate of sucrose containing probably 6 molecules of water. There appears to be no evidence which contradicts this conclusion.

\section{Summary.}

1. The average degree of hydration of sucrose in water solution at $0^{\circ}$ and at $30^{\circ}$ is calculated from the vapor pressures.

2. The theory is advanced that sucrose solutions are equilibrium mixtures of water, unhydrated sucrose and a single hydrate, and that the relative quantities of these substances are determined by the law of mass action.

3. This theory is tested by comparison of the experimental results with those calculated from the law of mass action. The agreement is fair for either a hexahydrate or a heptahydrate. The theory is also tested by a comparison of the activity of the sucrose calculated from its degree of hydration and that calculated by the Duhem-Margules equation. The results confirm those obtained by use of the law of mass action method.

4. The results agree with those obtained from the inversion of sucrose in the preceding paper.

AMHERST, MASSACHUSETTS. 